\section{"Time," a time-sharing moniter for the DEC LINC-8, PDP-8, and PDP-12}

\author{
PETER T. SCHWENN* \\ Department GYN/OB, University of Wisconsin Medical Center \\ Madison, Wisconsin 53706
}

A system is available for an $8 \mathrm{~K}$, or larger, Linc-8, PDP-8, or PDP-12 with RF/RS08 disk(s), to allow virtually simultaneous execution of any mix of PDP-8 and/or Linc programs. The programs need little or no modification to run within "Time," and up to eight may be in the mix at a given time. "Time" also incorporates a file system with access and write protection. The system is intended to be used with conversationally oriented programs. "Time" is in the public domain.

\section{INTRODUCTION}

"Time" is a computer program in PDP-8 code which allows the virtually simultaneous execution of (nominally) eight PDP-8 or Linc programs on an (8K) Linc-8. Versions will also be available for the PDP-8, PDP-12, and corresponding $4 \mathrm{~K}$ machines.

\section{FORM OF DESCRIPTION}

This description is organized to present the characteristics of Time to imaginary interested bystanders, e.g., computer system manager (the external view), the application programmer (internal view), and the systems programmer (system structure). Detailed limitations, in contrast to a dedicated PDP-8 or Linc, are presented also.

\section{EXTERNAL}

Time allows the concurrent use of a Linc-8 computer for several independent tasks, providing for a selection of tasks through a central point of control. A task can be any procedure coded as a Linc or PDP-8 (or both on the PDP-12) program, some limitations and some elaborations arising from the concurrent usage. A file system for Disk and LincTape, or DECTAPE, is available in the system which facilitates creation, modification, and deletion of program or data files and provides arbitrarily detailed access protection of files as desired. The file extends as a continuous stack from the first track of RF/RS08 disk to the last block of tape. A Linc program up to four banks in size which runs on any sort of Linc will run within Time with minimal limitations and virtually no modification. Any PDP-8 program of

*I thank Mr. Allen Puariea of the University of Wisconsin Medical Center's Medical Electronics Laboratory for ideas and suggestions on the software and for helping with the hardware. I also wish to thank Mr. William F. Carr and Dr. Ben M. Peckham of the Gynecology Department for providing motivation and support.
$4 \mathrm{~K}$ or less which uses only low-speed asynchronous I/O (e.g., TTY) will run within Time if its IOTs are replaced with calls to Time subroutines, or DEC's KT08 trap is installed. Generally, there is continuity of conversational $\mathrm{I} / \mathrm{O}$ without significant delay. A monitor-the central point of control-is part of Time, but its form is arbitrary: any possible monitor can be substituted without modification to the structure of Time. Time also allows for background tasks, and tasks to be initiated automatically at any desired time or repeated interval, such as accounting, weekly reports, Linc test programs, file up-dating or sorting programs, failsafe "file savers," etc. Line program development may be done within Time, and on-line programming may also be done through a programming system such as FOCAL. New tasks are easily added to the system.

\section{HARDWARE}

The system requires a DEC Linc-8 computer with at least $8 \mathrm{~K}$ of core memory, an RF/F808 disk system of any size, and one or more asynchronous communication channels, such as DEC PT08s. The system in the Gynecology Department of the University of Wisconsin Medical Center, where the system was developed, is an $8 \mathrm{~K}$ Linc- 8 with $500 \mathrm{~K}$ words of RF/RS08 disk, three PT08-FXs, four LincTape drives, a and analog data collection), an interblock zone interrupt (required if conversational communication is to proceed uninterrupted while LincTape is being read or written), and a microswitch console keyboard for use with the console display. More core, tape, disk, and channels may be added.

\section{MOTIVATION}

The system was developed to facilitate the independent use of a medical information system from several stations in an interregional 1 -sec clock (used for "time of day" program of the Wisconsin Regional Medical Program. This information system comprises several on-line conversational programs, including "interviewers" and more or less general retrieval programs to be used by physicians, paramedical personnel, and patients. The terminals are Teletypes (110 band) or CRT/keyboard terminals (up to 300 band) communicating asynchronously at local or remote sites, e.g., hospitals, over the dial telephone network. The analog facility of the Linc is handled in such a way as to allow for the development of natural-language interactive speech systems using telephones as terminals (so as to reduce interregional phone bills and terminal costs) and for analog-laboratory applications requiring long-term slow-speed and/or short-term high-speed analog sampling, e.g., clinical laboratories.

\section{INTERNAL VIEW}

The applications programmer has, from his point of view within Time, a $4 \mathrm{~K}$ PDP-8 (see limitations section) or a four-bank Line with a limitation that LMBs and UMBs must be replaced by their logical parallels in Time: OPRs $40-57$ and $60-77$, or be subject to check by Time, and the extension of Time OPRs as shown in Table 1 (restrictions follow "/").

In addition to the OPRs, MTP also has an elaborated function: In the mix table is a unit correspondence word $M_{5} M_{4} M_{1} M_{0}$; if an MTP is executed with unit $=n$, the true unit used will be $M_{n}$ where

$\begin{array}{rlr}M_{n}=0: & \text { disk unit } & 0 \\ 1 & & 1 \\ 2 & & 4 \\ 3 & & 5 \\ 4 & \text { LincTape } & 0 \\ 5 & & 1 \\ 6 & & 4 \\ 7 & & 5\end{array}$

in our University of Wisconsin Gynecology's configuration. In addition, a mix table option bit must be set to allow MTPs.

\section{SYSTEM ARCHITECTURE}

This can easily be provided by giving the form by chart of the file structure (Table 2), the mix table structure (Table 3 ), the disk/tape layout (Fig. 1), and the core layout (Fig. 2), and then the dynamics.

Figures 1 and 2 show the static structures of the system. Dynamically the system works as follows:

(1) Asynchronous $\mathrm{I} / \mathrm{O}$ is continuous inasmuch as no routine (except on-block LincTape logic) keeps the interrupt off for more than a millisecond; tape interrupts are recognized first, then the asynchronous $1 / O$ devices. The 
OPR $\quad 0-10 ; \quad$ Available (with some rore space to implement them):

11: Load PDP-8 prog. (Dir. \# IN A) and start at $[P+1]$ in Field $1 /$ this can be executed only by the monitor "TSLNK." this can be executed only by the monitor "TSLNK."

12: MBS to Linc A: "A(L)"

13: JMS [A(L)] / appropriate mix table option bit must be set

14: TYP (If $A(L)=4000$ Buffer is cleared

15: KBD $35: \mathrm{KBD}$ ।

16: RSW 17: LSW

20: Disable swapping

RETURN (illegal) $\rightarrow P+1$

Else $\rightarrow p+2$, appropriate mix bit set and no other program in mix disallows

21: Reenables swapping

22: $\quad[P+1]-4000: \quad$ disconnect $m$

0001: log me out

4001: both

0: $\quad$ connect me

(will become call out [number: $A(L)$ ] with auto dial out equipment)

23: Swap me out now, bring me back in on my turn

24: Flag SET/CLEAR/SKIP/LOAD

P + 1- ONOM

$\mathrm{P}+1-\mathrm{ON} O \mathrm{~N}=3: 0$
$\mathrm{O}=\mathrm{N}$

$[P+2] \rightarrow$ flag $M, R \rightarrow P+3$

1:

clear flag $M, R \rightarrow P+$

set flag $M, R \rightarrow P+2$

If flag $M \operatorname{set}(\neq 0), R \rightarrow P+3$, else $R \rightarrow P+2 \epsilon$.g.,

$\mathrm{O}=\mathrm{N}=7:$ Flag Number skip on flag $M$

25: $\quad$ Time internal information set/retrieve $A=L M N N$ on entry

$\begin{array}{rlll}L: & \neq 0: & \text { Set (Info. from } P+1, \text { return to } P+2) \\ & =0: & \text { Retrieve }\end{array}$

M: Mix number $(0=$ "my" mix number $)$

NN: Which parameter to set or retrieve

00: NINMIX/CTMIXN 6 bits per

01-20: Corresponding mix table word

21-77: available

/ appropriate mix table option bit must be set in order to "set," no restriction on retrieve

26: Zero my disconnect clock

27: Number of seconds since Midnight Dec 31,1900 to $P+1,2,3$, return to $P+4$

30: Open or close File / appropriate mix bit(s) must be set A on entry:

bit $0 \quad 1$-close

0-open

1: $\quad 1$-password present at $P+1,2$ as 4 LincCodes

0 -no password present

2: 1 -file options, key for file creation present at $\mathrm{P}+1$ or $\mathrm{P}+3$

0 -use standard options

3: $\quad 1$-establish file if it doesn't exist

0-don't

4-8: not used

9.11: logical file number 0-7

Function: if legal, open or close the file and return at args +2 with $A$ containing the length of the file: if illegal return at args +1 with $A$ containing

$\begin{array}{ll}\text { 0000: } & \text { password fails } \\ 7777: & \text { access otherwise denied } \\ \text { 4000: } & \text { no such file }\end{array}$

31: Read or Append or Delete or Write within File. / appropriate mix bits(s) must be set

$P+1$ on entry: $111 \mathrm{omm}$ mmn nnn: LMN

$L$ : $\quad$-read. 1-append, 2-delete, 3-write

L: $\quad$ O-read, 1-appen

$\mathrm{N}$ : begin at quarter $\# \mathrm{~N}$ relative to quarter 0 in my $L M B$

$\mathrm{P}+2$ on returm: initial block number in file

$P+3$ : error return $A$ :

o-unopened file

1 -irrecoverable error

2-too many blocks requested

$P+$ 4: normal return $A$ :

normal retur

1-disk / tape boundary crossed

32: Directory read (not yet implemented)

33: Initiate background program (not yet implemented)

34: Dump background or periodic program output (not yet implemented)

36: Intercom: communicate with another terminal (not yet implemented)

37: Analog data request (not yet implemented)

4N: Logical UMB N / legality is checked

6N: Logical LMB N/dito

LincTape limits continuous $1 / O$ to $<25$ characters/sec during its operation.

(2) A "rotating" queue is the basis of the timesharing scheduler. The queue is "rotated" by firing and subsequently recognizing the restart-delay clock of the Linc-8. If a slot in the queue contains a program not paused on $1 / O$, it is brought into
Meta Directory-fixed at block 0034, 4 blocks long. 4 words/entry

Word 1: $t_{0} t_{1} t_{2} t_{3} d d$ ddd ddd

Type $t_{0}:$ 1-program

$t_{1}: 1-\mathrm{read} / \mathrm{write}$

0-read only

$t_{2}:$ 1-movable in file

0-fixed (e.g., position of directory)

$t_{3}:$ 1-password required 0-not

Directory number: dd ddd ddd (0-255)

Word 2,3,4: 6 character name

Directory-fixed at block $0240,20_{8}$ blocks long, $20_{8}$ words/entry

Word 1: Unit \#/Block \# (0/000-7/777)

2: $n / /$ length in blocks-1

$\mathrm{n}$ : $\quad 0-\mathrm{RSO8}$

1-LincTape

2-RK08

3.4: 4 character password

$5,6,7,10$ : access protection "description: PROGRAM

Word 11: Starting locations

12,13: Mix options words

14.15: Unit requirements

16: Core requirements

OTHER

11-16: free

Table 3

Mix Table Structure

$10_{8}$ "slots." $20_{8}$ words / slot: (program mix entry)

Word 1: Station \#

2: bit $0: \quad$ Link bit

3,4,5: Terminal ty $p$

8: Pause bit

9,10,31: Channel number

3: Program counter

4: Accumulator

5: $\mathbf{Z}$ for Linc, IB for PDP-8

6: LMB/UMB

7: Time program was started (least sig. clock digits)

10: Directory number

11,12: Option words

Word 1 / Word 2

bit: 0 : Cannot tolerate swap inhibit no passwords needed

1: Convert input to LincCode / can create files

2: Echo chan. bit 0 / can delete

3: Echo chan. bit 1 / can write

4: Echo chan. bit $2 /$ can append

5: Can execute MTPs \& OPRs $20.26 /$ can read

6: Background program / free

7: Can execute OPR 25 in set mode / free

8: Can execute OPRs 11 and 13 free

9: Program is restored after use / Unrestricted UMBs, LMBs

10: One user program / Allow intercom request break in

11: PDP-8 program / Periodic program

Word 13: Unit correspondence

14: Swap code

15: Core indicator

16: User \#

17: Available

Field 1 after saving the current program in its slot. Versions of time for the PDP-8 or -12 will require the use of a device other than the restart delay; on the PDP-12 the KW12 clock suffices, but the PDP-8 would require a "fireable," interrupting clock as a hardware addition.

(3) PDP-8 programs are 


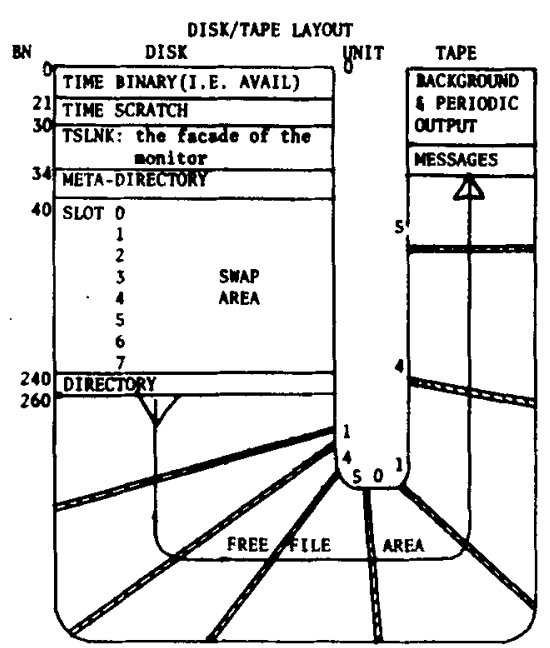

Fig. 1. Disk/tape layout of the system architecture.

distinguished by reference to the mix table and by noting on interrupt whether the field of origin was not equal to 0 -alternatively by KT 08 or $\mathrm{KT} 8 / \mathrm{I}$ trap hardware. PDP-8 $\mathrm{I} / \mathrm{O}$ is handled by jumps from the PDP.8 program as follows:

$\left.\begin{array}{l}\text { CIF } \\ \text { IOF } \\ \text { JMS; * + 1 } \\ \text { address } \\ \text { return }\end{array}\right\}$ or $\begin{cases}\text { IOF } & \\ \text { IOFS; } & \text { (address in } \\ \text { return } & \text { page zero) }\end{cases}$

\section{LIMITATIONS}

The limitations given are those relative to a single-user, dedicated Linc-8, except as noted:

(1) Real time is distorted by swapping and by interrupts unrelated to a given user's task; this can in part be avoided by inhibiting the swapping temporarily by OPR 20. Analog sampling at rates up to $20 \mathrm{~Hz}$ can be had via OPR 37 with minimal clock time distortion, depending on the particular rate desired, and up to $\sim 100 \mathrm{KHz}$ with swapping inhibited.

(2) Each user has available as a medium a terminal such as TTY rather than the computer console and TTY. However, "sense switches" are available through OPR 24.

(3) Unless DECs KT08 or similar IOT + HLT trapping hardware is present, no untested PDP-8 programs can be allowed safely in the mix.

(4) Hardware to cause interrupt on UMB or LMB, a trivial and inexpensive modification, must be present to provide total protection against Linc-user programming errors; that is, if Linc program development is to be done within Time.

(5) Since there is no priority interrupt system, the number of "concurrent" terminals is limited when PT08s are used as I/O channels. I do not know the limit yet. (DEC has equipment to mitigate.)

(6) (General limitation on Linc-8.) Asynchronous $\mathrm{I} / \mathrm{O}$ must be limited to about 20 characters/sec per terminal during LincTape use. This affects only terminals other than TTYs.

\section{SUBSYSTEMS NOW AVAILABLE}

INTVW, SUMRY, and FND are three programs for information storage, retrieval, and analysis (FND is Richard Harshman's FIND somewhat anglicized). Other subsystems now available are INTERCOM for interterminal communication and DEC's FOCAL, and, of course, any Linc or PDP-8 program within the limitations noted above.

\section{SUBSYSTEMS COMING}

These include: DEC's BASIC, a more fully English FIND; DEC's FORTRAN, a message and reminder system; PROGRAM, an editor and assembly system for Linc
CORE LAYOUT

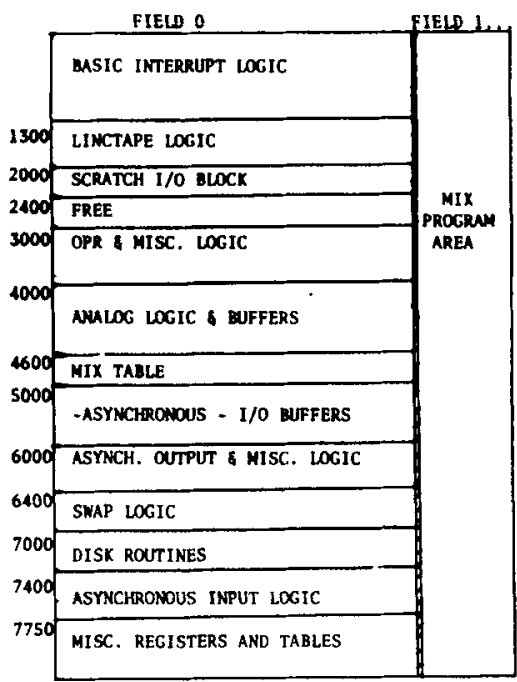

Fig. 2. Core layout of the system architecture.

programming; LINC/8, an assembler for mixed PDP-8 and LincCode; AUDINT, a data-gathering system requiring only a dial telephone as remote terminal; and BACKGROUND, for executing noninteractive sets of programs.

OTHER VERSIONS OF TIME

Versions are currently being written for $\geqslant 8 \mathrm{~K}$ PDP-8 and PDP-12s, both for those with hardware trap of IOTs and HLTs and those without. A version could be written for $4 \mathrm{~K}$ Linc- 8 allowing two-bank Linc programs only and very few of the extra OPRs. Versions for $12 \mathrm{~K}$ and greater will lose much less to overhead than will the $8 \mathrm{~K}$, though this is relatively unimportant for some large groups of conversational, noncomputational tasks. 